\title{
Insetos galhadores associados a duas espécies de plantas invasoras de áreas urbanas e peri-urbanas
}

\author{
Genimar R. Julião ${ }^{1}$, G. Wilson Fernandes²,3, Daniel Negreiros², Lúcio Bedê4, \& Raquel C. Araújoº
}

'Doutorado em Ecologia-Instituto Nacional de Pesquisas da Amazônia, V8, DCEC, CP 478, 69011-970 Manaus-AM.
2Ecologia Evolutiva de Herbívoros Tropicais-DBG, ICB, Universidade Federal de Minas Gerais, CP 486, 30161-970 Belo Horizonte-MG.
${ }^{3}$ gwilson@icb.ufmg.br
${ }^{4}$ Doutorado em Ecologia e Manejo da Vida Silvestre-DBG, ICB, Universidade Federal de Minas Gerais, CP 486, $30161-970$ Belo Horizonte-MG.

\begin{abstract}
Aвstract. Galling insects associated with two species of ruderal plants in urban and peri urban areas. Insects have been considered as important bioindicators of environmental changes and habitat quality. In spite of its sessile habit, easy localization, abundance and host specificity, insects that induce galls have not been utilized in studies of this nature. It was investigated the suitability of gall-inducing insects associated to two ruderal host plant species (Baccharis dracunculifolia and Vernonia polyanthes: Asteraceae) as bioindicators of habitat quality. The following questions were addressed: (i) is gall-inducing insect diversity influenced by different types of land use?; (ii) are the responses of galling insect communities different between host plants?; (iii) how does the biotic and physical features of the biotope influence the gall-inducing insect diversity? It was found 6,226 galls, belonging to six galling insect species on $V$. polyanthes and 11 galling species on $B$. dracunculifolia. No difference was found in galling species richness among land use types. Nevertheless, gall-forming insect abundance was statistically different among the biotopes studied. Insect galls were more numerous in biotopes with lower urbanization levels. Gall abundance showed a strong and positive relationship with the percentage of vegetal cover. Gall-forming insect communities on both host species showed differential responses to the different land use types. The results suggest that three factors may be involved with galling insect diversity in urban areas: (i) habitat structure in the biotope; (ii) resource abundance (host plant abundance and distribution); and (iii) frequency and intensity of management in reserves, parks, city squares, wastelands found at a given urban area.
\end{abstract}

KeYwORDs. Bioindicator species; biotopes; host plants; insect galls; urban-rural gradient.

REsumo. Insetos galhadores associados a duas espécies de plantas invasoras de áreas urbanas e peri-urbanas. Os insetos têm sido considerados importantes indicadores de mudanças ambientais e da qualidade de habitats. Apesar de seu hábito séssil, fácil visualização, abundância, e especificidade de hospedeiro, insetos indutores de galhas não têm sido utilizados em estudos desta natureza. Neste estudo foi investigado o uso potencial de insetos galhadores associados a duas espécies de plantas hospedeiras ruderais (Baccharis dracunculifolia e Vernonia polyanthes: Asteraceae) como bioindicadores da qualidade de habitats. Procurou-se responder às seguintes questões: (i) A diversidade de insetos galhadores é afetada pelo tipo de uso e ocupação da paisagem (solo)?; (ii) A resposta das comunidades de insetos galhadores difere entre as duas espécies de plantas hospedeiras?; (iii) A diversidade de insetos galhadores é influenciada por características bióticas e físicas dos biótopo urbanos? Foram coletadas 6.226 galhas, pertinentes a 6 espécies de insetos galhadores associados à $V$. polyanthes e 11 espécies associadas à $B$. dracunculifolia. Não foi encontrada nenhuma diferença na riqueza de insetos galhadores entre os biótopos amostrados. No entanto, a abundância de insetos galhadores apresentou diferenças significativas quanto ao tipo de uso e ocupação da paisagem. As galhas foram mais numerosas em biótopos menos urbanizados, sendo observado uma relação forte e positiva com a porcentagem de cobertura vegetal do biótopo. As comunidades de insetos galhadores de ambas as espécies de plantas hospedeiras apresentaram respostas diferenciais quanto ao tipo de uso da paisagem. Os resultados sugerem três fatores que podem estar envolvidos com a diversidade de insetos galhadores em áreas urbanas: (i) estrutura dos habitats num biótopo; (ii) abundância e distribuição espacial do recurso planta hospedeira e; (iii) freqüência e intensidade do manejo de reservas, parques, jardins e terrenos baldios de uma dada área urbana. Constatou-se ainda que áreas verdes em espaços urbanos são de fundamental importância na manutenção da diversidade de insetos.

Palavras-Chave. Bioindicadores; biótopos; galhas de insetos; gradiente rural-urbano; plantas hospedeiras.

Ecossistemas urbanos consistem em áreas sob intensa $\mathrm{e}$ constante atividade humana, associada a altos índices populacionais, presença de pólos industriais, centros comerciais e resquícios de vegetações nativas e/ou alteradas. O processo de urbanização envolve a substituição de áreas nativas por várias formas de uso antropogênico da paisagem (McIntyre et al. 2001). Nesse contexto, é necessário distinguir os principais componentes da urbanização que possibilitem verificar padrões espaciais na estrutura e função de sistemas ecológicos, tanto em abordagens populacionais, de comunidades e paisagens. $\mathrm{O}$ gradiente rural-urbano mostrase, então, de grande utilidade em pesquisas ecológicas, pois permite avaliar o grau de influência de uma alteração espacial decorrente da urbanização e seus efeitos ambientais e ecológicos (McDonnell \& Pickett 1990).

Insetos são considerados excelentes bioindicadores de mudanças ambientais (poluição do ar e da água) e da qualidade do ambiente. Estudos de suas comunidades podem ser 
aplicados no planejamento, desenho e manejo urbano (Zapparoli 1997), monitoramento ambiental e práticas conservacionistas (Samways 1994, Fernandes et al. 1995, Olivier et al. 1998, Taylor \& Doran 2001). Todavia, devido à complexidade da taxonomia de artrópodes, mais especificamente insetos, o número de estudos utilizando esses grupos em avaliações ambientais tende a ser limitado (McIntyre et al. 2001). Dessa forma, a utilização de morfoespécies tem sido amplamente discutida como forma de solucionar esta limitação (Disney 1986) e aplicada com êxito (Olivier \& Beattie 1996, Pik et al. 1999), porém pouco difundida.

Contudo, insetos que induzem galhas podem constituir excelente ferramenta para estudos ambientais por serem sésseis, de fácil localização, abundantes e hospedeiroespecíficos (Fernandes et al. 1995, Lara \& Fernandes 1996). Bagatto \& Shorthouse (1991) utilizaram galhas de insetos para avaliar o impacto de siderúrgicas próximas a Sudbury, Ontario (Canadá). Altas concentrações de cobre e níquel foram encontradas nas galhas, comparado a outros tecidos vegetais da planta hospedeira. Foi verificado ainda que as concentrações desses metais nas plantas e no tecido das galhas diminuía com a distância das siderúrgicas. Galhas induzidas por um fungo em Acacia karroo (Leguminosae) apresentaram menores valores de ocupação de galhas, densidades de larvas e riqueza de espécies de lepidópteros associados, em sítios urbanos mais impactados (McGeoch \& Chown 1997). Todavia, são poucos ainda os estudos desta natureza, não obstante a diversidade e ampla ocorrência de galhas de insetos.

Neste trabalho, investigou-se as comunidades de insetos galhadores associadas a duas espécies de plantas ruderais em áreas urbanas e peri-urbanas, com o objetivo de avaliar o seu uso potencial na inferência da qualidade ambiental de biótopos urbanos. Em um gradiente rural-urbano, biótopos urbanos podem ser definidos como unidades do espaço com características comuns, referentes à estrutura (aspectos bióticos e físicos) e às formas / intensidade de uso incidentes. O conjunto de características e descrições comuns de um biótopo são denominadas tipologias (Bedê et al. 1997). Neste estudo, a identificação de tais biótopos foi baseada na proposta de Sukopp \& Weiler (1988), com base nos critérios referidos acima. Dessa forma, procurou-se responder às seguintes perguntas: (i) diferentes tipologias urbanas influenciam a diversidade de insetos galhadores?; (ii) há diferenças na resposta das duas comunidades de insetos galhadores, em termos de riqueza de espécies e abundância, ao longo do gradiente urbano? e (iii) a diversidade de insetos galhadores se relaciona com as características físicas e bióticas dos biótopos?

\section{MÉTODOS}

Áreas de estudo. O estudo foi desenvolvido na região metropolitana de Belo Horizonte, MG, Brasil, entre maio e setembro de 1994. As amostragens foram feitas em 24 localidades agrupadas em 7 tipologias de biótopos urbanos
(Tabela I), caracterizados com respeito ao grau de impermeabilização, tipos predominantes de edificações e forma/ intensidade de manejo das áreas livres (Bedê et al. 1997): áreas rurais (Rural); áreas verdes (Áreas verdes urbanas); biótopos com predominância de residências unifamiliares (Casas); biótopos compostos por mosaicos de residências unifamiliares, prédios residenciais unifamiliares e comerciais (Prédios e Casas); biótopos com predominância de prédios comerciais (Prédios); favelas e áreas de uso industrial.

Duas variáveis (características físicas e bióticas) foram utilizadas para caracterizar bairros pertinentes às tipologias mencionadas (Tabela I; Prefeitura de Belo Horizonte, 1994): Percentagem de Cobertura Vegetal $(\% \mathrm{CV})$ e Percentagem de Ruas Pavimentadas, como parâmetro indicador do grau de permeabilidade do solo (\%RP). Para o bairro Eldorado, pertinente ao município vizinho de Contagem, foram utilizados valores médios destas variáveis para bairros circunvizinhos de mesma tipologia, inseridos no município de Belo Horizonte.

Amostragem. A coleta de dados foi realizada ao longo de três percursos aleatórios, cada um com duração de uma hora (veja Price et al. 1998), em cada uma das localidades. Foram realizadas, ao todo, 54 caminhadas em 18 localidades. Durante as caminhadas houve busca intensa pelas espécies hospedeiras. No entanto, seis localidades não apresentaram as espécies de plantas enfocadas neste estudo (ver Tabela I). Todas as plantas encontradas tiveram sua riqueza e abundância de espécies de insetos galhadores avaliadas. Além disso, as galhas de insetos foram caracterizadas quanto à sua morfologia externa, pubescência, cor, tipo de ocorrência (isolada ou agrupada) e ilustradas (veja Julião et al. 2002).

Plantas hospedeiras. Foram selecionadas duas espécies ruderais, de ampla distribuição no ambiente urbano em questão:

Baccharis dracunculifolia DC - alecrim-vassoura (Asteraceae). Arbusto dióico, perene, lenhoso, com altura de 2 a 3 metros, nativo do Brasil, comum em áreas de cerrado, pastagens abandonadas e áreas em processo de sucessão. A floração desta espécie ocorre após o período de chuvas (Boldt 1989, Espírito-Santo \& Fernandes 1998). Fernandes et al. (1996) relataram uma rica fauna de insetos galhadores associada ao gênero Baccharis Linnaeus, ocorrendo cerca de 17 tipos de galhas associados a $B$. dracunculifolia.

Vernonia polyanthes (Spreng.) Less - assa-peixe (Asteraceae). É uma espécie invasora de áreas cultivadas e sob alguma forma de distúrbio (Leitão Filho et al. 1972). Possui várias espécies de insetos galhadores associados, mas estudos sobre esta comunidade de insetos são escassos (veja Andrade et al. 1995 e Silva et al. 1996).

Análise dos dados. Os dados coletados neste estudo não apresentaram distribuição normal, em sua maior parte. Assim, optou-se pela análise não-paramétrica e a não utilização de qualquer artifício para a normalização dos dados (ver Samways 

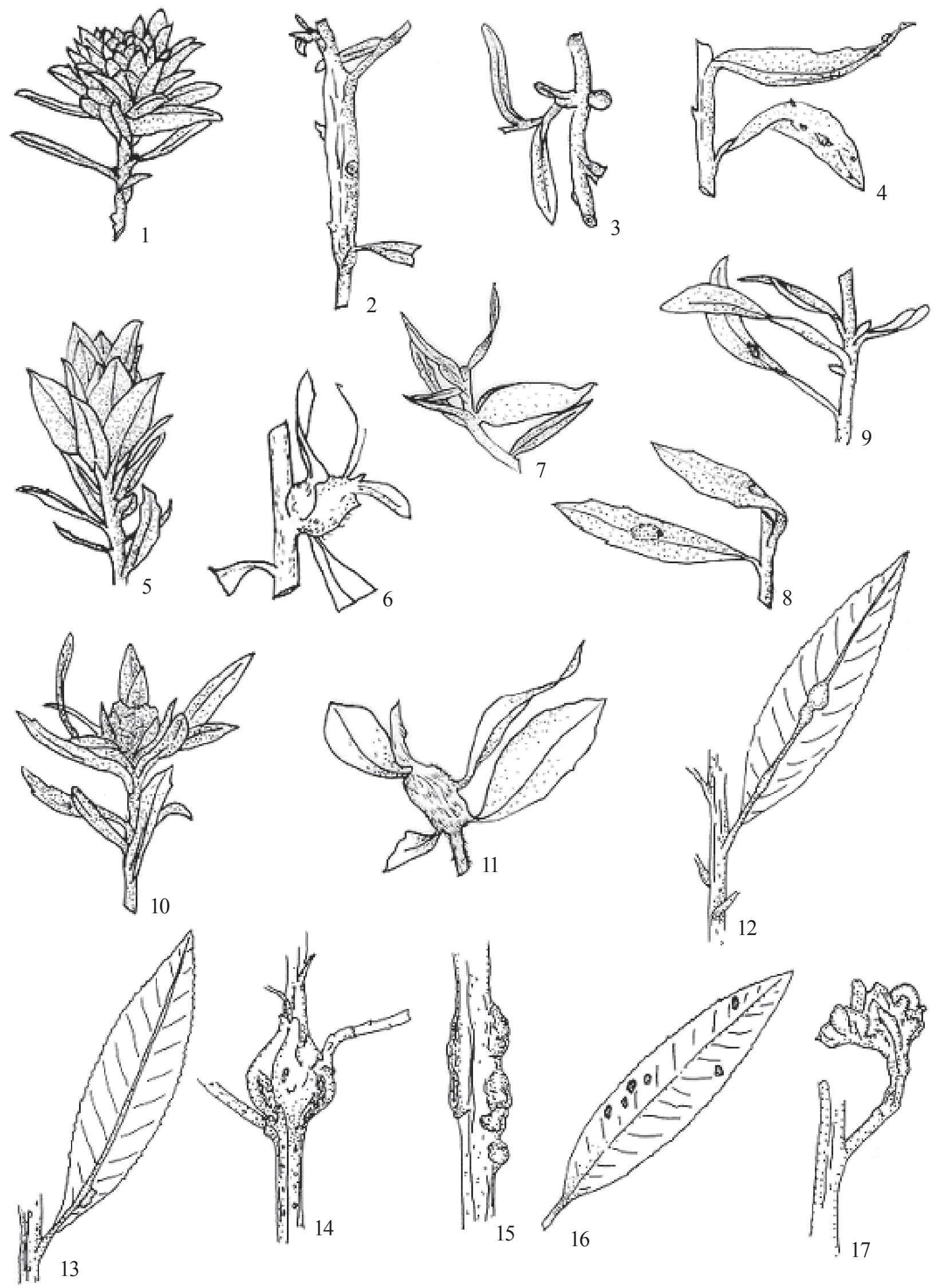

Figs. 1-11. Morfo-tipos de galhas de insetos associadas a Baccharis dracunculifolia DC (Asteraceae) (modificado de Fernandes et al. 1996). Figs. 12-17. Morfo-tipos de galhas de insetos associadas a Vernonia polyanthes (Spreng.) Less (Asteraceae). 

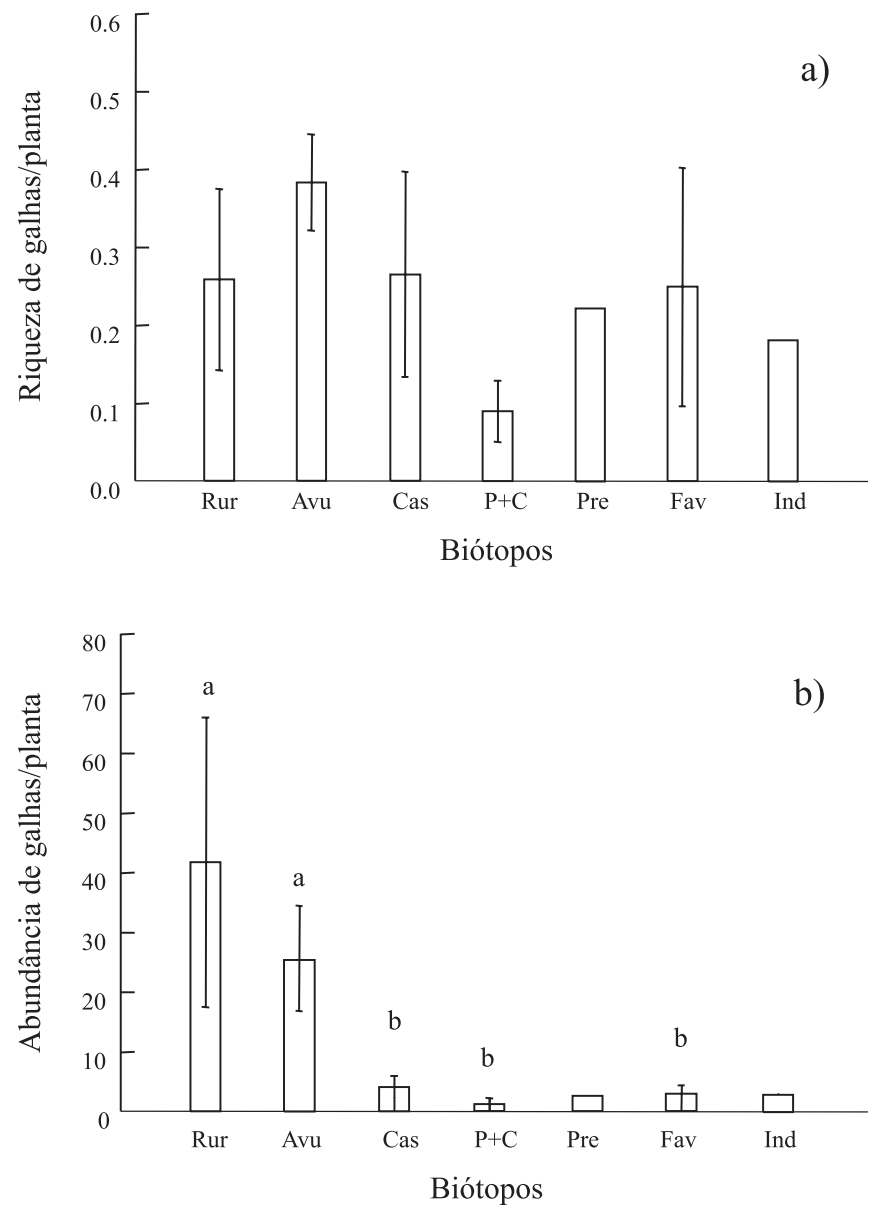

Fig. 18a,b. Riqueza (a) e abundância (b) de insetos galhadores por planta hospedeira nos biótopos urbanos de Belo Horizonte, MG. (Rur: Rural; Avu: Áreas verdes urbanas; Cas: Casas; P+C: Prédios e Casas; Pre: Prédios; Fav: Favelas e Ind: Industrial).

\& Steytler 1996). Para avaliar como as duas espécies de plantas hospedeiras respondem aos diferentes graus de urbanização, foi realizado o teste Kolmogorov-Smirnoff com os valores de abundância de galhas das duas espécies nos diferentes biótopos, assim como comparações visuais das curvas de riqueza de galhas. Tanto a riqueza quanto a abundância de galhas das duas plantas hospedeiras foram agrupadas para uma melhor visualização da influência do grau de urbanização na comunidade de insetos galhadores. Além disso, estes valores foram divididos pelo número de plantas amostradas e pelo número de unidades amostrais (localidades), de forma a subtrair o efeito de diferentes esforços amostrais nos biótopos. O teste Kruskal-Wallis foi usado para avaliar diferenças na abundância e riqueza de galhas entre biótopos. O índice de Shannon-Winner $(\mathrm{H})$ e Equitabilidade (Eh) foram calculados a partir da abundância e riqueza de galhas de insetos em Baccharis dracunculifolia e Vernonia polyanthes, como medida da diversidade de galhas em cada biótopo. Para se avaliar a relação entre o grau de urbanização e a abundância e riqueza de galhas, foram realizadas matrizes de correlação de
Pearson com as variáveis \%CV, \%RP, abundância e riqueza de galhas das localidades amostradas (Zar 1996).

\section{RESULTADOS}

Foram amostradas 6.226 galhas, sendo 3.540 (57\%) galhas distribuídas entre as 6 morfo-espécies de insetos galhadores de Vernonia polyanthes e 2.686 (43\%) pertinentes às 11 morfoespécies associadas a Baccharis dracunculifolia (Figuras 1 a 17 - Tabela II). A morfo-espécie 3 (Figura 14) da planta Vernonia polyanthes é induzida por Tomoplagia rudolphi Lutz \& Costa Lima (Diptera, Tephritidae), havendo relatos de sua biologia (Andrade et al. 1995) e da relação com sua planta hospedeira (Silva et al. 1996).

As menores abundâncias relativas de galhadores associados a $B$. dracunculifolia foram observadas nas morfoespécies G3, G4, G5, G9, G10, G11, sendo as galhas G1, G2, G3 e G9 as que ocorreram em maior número de localidades. Os maiores valores de abundância relativa foram da morfo-espécie $6(\mathrm{G} 6,0.932)$ na localidade Zoológico e da morfo-espécie 2 (G2, $0.875)$ no bairro Sion. Os menores valores foram da morfoespécie $6(\mathrm{G} 6,0.006)$ na localidade Horto e da morfo-espécie 1 (G1, 0.008) no Zoológico (Tabela III). Cerca de 50\% das 2.686 galhas amostradas em $B$. dracunculifolia foram causadas pelas morfo-espécies G7 e G8.

As morfo-espécies de galhadores de $V$. polyanthes mais freqüentes nos locais amostrados foram G1, G3 e G6, sendo G2, G4 e G5 as morfo-espécies que apresentaram as menores abundâncias relativas nos locais amostrados. Os maiores valores de abundância relativa foram da morfo-espécie 1 (G1, $0.808)$ na localidade Trevo e da morfo-espécie 3 (G3, 0.818) no bairro Eldorado. Os menores valores foram da morfo-espécie 2 (G2, 0.002) na localidade Estação Ecológica e da morfo-espécie 4 (G4, 0.003) na localidade Zoológico (Tabela IV). Das 3.540 galhas amostradas em $V$. polyanthes, cerca de $60 \%$ pertenciam a morfo-espécie G1.

Diversidade de galhadores nos diferentes biótopos. A riqueza de galhadores por planta hospedeira não variou entre os biótopos (Kruskal-Wallis $=8.806$, d.f. $=6, p=0.185)$. Contudo, foi observada uma diferença marginalmente significativa na abundância de galhadores por planta hospedeira entre os biótopos (Kruskal-Wallis $=12.526$, d.f. $=6, p=0.051)$. Verificouse um maior número de galhas nos biótopos com menor intensidade de ocupação (biótopos de áreas rurais e áreas verdes urbanas), enquanto um declínio gradual no número de indivíduos ocorreu na porção final do gradiente rural-urbano, havendo menos indivíduos nos biótopos Prédios e Casas, Prédios, Favelas e Industrial (Figura 18a,b). As plantas hospedeiras de insetos galhadores do biótopo Rural apresentaram, aproximadamente, 10 vezes mais galhas que as plantas do biótopo Casas, 35 vezes mais galhas que o biótopo Prédios e Casas e 14 vezes mais que o biótopo Favelas. Já o biótopo Áreas verdes urbanas apresentou, em média, 6 vezes mais galhas que o biótopo Casas, 21 vezes mais galhas que o biótopo Prédios e Casas e 8 vezes mais que o biótopo Favelas. 
Tabela I. Biótopos e suas características, localidades amostradas em Belo Horizonte, MG e valores de porcentagem de Cobertura Vegetal (\%CV) e porcentagem de Ruas Pavimentadas (\%RP) de cada local (Prefeitura de Belo Horizonte, 1994).

\begin{tabular}{|c|c|c|c|c|}
\hline Biótopos & Caracterização & Localidades & $\% \mathrm{CV}$ & $\% R P$ \\
\hline \multirow[t]{4}{*}{ Rural } & \multirow{4}{*}{$\begin{array}{l}\text { Áreas onde persiste uso rural secundário, com ocorrência } \\
\text { pontual de áreas remanescentes do antigo contexto rural. }\end{array}$} & Baleia & 58,91 & 36,74 \\
\hline & & Médio Barreiro * & - & - \\
\hline & & São Benedito & 51,27 & 64,95 \\
\hline & & Trevo & 61,32 & 43,49 \\
\hline \multirow{5}{*}{$\begin{array}{l}\text { Áreas verdes } \\
\text { urbanas }\end{array}$} & \multirow{5}{*}{$\begin{array}{l}\text { Áreas verdes e parques públicos / áreas de lazer. Possuem } \\
\text { poucas edificações e pequena superfície tomada por ruas e } \\
\text { caminhos. Presença de formações vegetacionais nativas, em } \\
\text { estágios sucessionais diversos. }\end{array}$} & Lagoa do Nado * & - & - \\
\hline & & Horto & 36,89 & 76,02 \\
\hline & & $\begin{array}{l}\text { Estação Ecológica } \\
\text { (UFMG) }\end{array}$ & 68,00 & 100,00 \\
\hline & & Zoológico & 53,37 & 96,13 \\
\hline & & Ursulina * & - & - \\
\hline \multirow[t]{4}{*}{ Casas } & \multirow{4}{*}{$\begin{array}{l}\text { Áreas de uso predominantemente residencial, com edificações } \\
\text { unifamiliares de } 1 \text { a } 3 \text { andares e áreas livres privativas. Poucas } \\
\text { edificações de uso comercial. Ajardinamento } \\
\text { predominantemente ornamental. }\end{array}$} & Serra & 37,31 & 82,53 \\
\hline & & Bandeirantes & 58,46 & 55,91 \\
\hline & & Castelo & 51,44 & 83,96 \\
\hline & & Prado & 30,41 & 91,57 \\
\hline \multirow[t]{4}{*}{ Prédios e casas } & \multirow{4}{*}{$\begin{array}{l}\text { Mosaicos compostos por prédios de uso residencial e } \\
\text { comercial e residências unifamiliares com áreas livres } \\
\text { privativas. Ajardinamento predominantemente ornamental. }\end{array}$} & Funcionários & 34,08 & 100 \\
\hline & & Caiçara & 36,69 & 86,69 \\
\hline & & Sion & 36,60 & 90,16 \\
\hline & & Coração Eucarístico & 38,50 & 99,92 \\
\hline \multirow[t]{2}{*}{ Prédios } & \multirow{2}{*}{$\begin{array}{l}\text { Áreas com predomínio de prédios de uso comercial, com } \\
\text { elevado grau de verticalização e adensamento. Áreas livres } \\
\text { reduzidas e fortemente impermeabilizadas. }\end{array}$} & Eldorado & 37,66 & 82,00 \\
\hline & & Centro * & - & - \\
\hline \multirow[t]{4}{*}{ Favelas } & \multirow{4}{*}{$\begin{array}{l}\text { Predominam pequenas residências unifamiliares, de } 1 \text { a } 2 \\
\text { andares, de disposição desordenada e adensada. Áreas } \\
\text { privativas livres muito reduzidas, com ajardinamento } \\
\text { predominantemente utilitário. Grau de impermeabilização das } \\
\text { áreas livres e caminhos variável. }\end{array}$} & Cafezal (Serra) & 40,41 & 43,45 \\
\hline & & Vera Cruz* & - & - \\
\hline & & São José * & - & - \\
\hline & & Nova Pampulha & 47,09 & 57,74 \\
\hline Industrial & $\begin{array}{l}\text { Indústria pesada, fábricas e depósitos. Áreas livres fortemente } \\
\text { inpermeabilizadas. Ajardinamentos reduzidos, de ocorrência } \\
\text { pontual e finalidade ornamental. }\end{array}$ & Bairro das Indústrias & 26,77 & 100 \\
\hline
\end{tabular}

*Obs: Nas localidades marcadas em asteriscos não foram encontradas as duas plantas hospedeiras visadas neste estudo. São elas: Médio Barreiro, Lagoa do Nado, Ursulina, Centro, Favela do Vera Cruz, Favela do São José.

Baccharis dracunculifolia vs. Vernonia polyanthes. As comunidades de galhadores associadas a $B$. dracunculifolia e a $V$. polyanthes apresentaram respostas similares, com relação ao parâmetro abundância $(\mathrm{p}=0.098$; Figura 19) nos diferentes biótopos amostrados. A abundância de galhas em $B$. dracunculifolia e em $V$. polyanthes foi menor nos biótopos situados no extremo do gradiente rural-urbano caracterizado pela maior intensidade de ocupação (Prédios, Favelas e Industrial). A riqueza de galhas em Baccharis dracunculifolia apresentou, similarmente, o mesmo padrão, sendo maior nos biótopos menos intensamente urbanizados, enquanto a riqueza de galhas em $V$. polyanthes não variou ao longo do referido gradiente (Figura 20).

Diversidade de insetos galhadores nos biótopos. Os maiores valores de diversidade e equitabilidade observados na comunidade de insetos galhadores associados a $B$. dracunculifolia ocorreram nos biótopos Casas $>$ Áreas verdes urbanas $>$ Rural. Essa tendência não foi observada na comunidade associada a $V$. polyanthes, com referência aos valores de diversidade e equitabilidade, no gradiente referido. A variação nos valores do índice de diversidade foi Casas $>$ Áreas verdes urbanas $>$ Favelas $>$ Industrial $>$ Prédios e casas $>$ Rural $>$ Prédios, enquanto os valores de equitabilidade seguiram a seguinte ordem: Casas $>$ Favelas $>$ Áreas verdes urbanas $>$ Prédios e casas $>$ Prédios $>$ Industrial $>$ Rural (Tabela V).

Características do biótopo e diversidade de galhadores. A Percentagem de Cobertura Vegetal (\%CV) apresentou uma forte relação positiva com a abundância de galhas $(r=0.80 ; p<0.05)$. Esta relação foi moderada e positiva com a riqueza destes organismos nas localidades amostradas $(\mathrm{r}=0.64 ; \mathrm{p}<0.05)$. Já 
Tabela II. Descrição das morfo-espécies de galhas induzidas em Baccharis dracunculifolia e Vernonia polyanthes amostradas em biótopos urbanos de Belo Horizonte, MG.

\begin{tabular}{|c|c|c|c|c|c|c|c|}
\hline \multirow{2}{*}{$\begin{array}{l}\text { Planta } \\
\text { Hospedeira }\end{array}$} & \multirow{2}{*}{$\begin{array}{l}\text { Morfo- } \\
\text { espécie }\end{array}$} & \multirow{2}{*}{ Taxa do galhador } & \multicolumn{4}{|c|}{ Descrição da gallha } & \multirow{2}{*}{ Figuras } \\
\hline & & & Órgão & Forma & Cor & Pubescência & \\
\hline Baccharis & 1 & Cecidomyiidae & Botão apical & roseta & verde & Glabra & Fig. 1 \\
\hline dracunculifolia & 2 & Lepidoptera & Ramo & elipsóide & verde & Glabra & Fig. 2 \\
\hline \multirow[t]{9}{*}{$\mathrm{DC}$} & 3 & Cecidomyiidae & Botão foliar & esferóide & verde & Pilosa & Fig. 3 \\
\hline & 4 & Cecidomyiidae & Folha & cônica & verde & Glabra & Fig. 4 \\
\hline & 5 & Asphondylia sp. & Botão apical & roseta & verde & Glabra & Fig. 5 \\
\hline & 6 & Lepidoptera & Ramo & globóide & verde & Pilosa & Fig. 6 \\
\hline & 7 & $\begin{array}{l}\text { Neopelma baccharidis } \\
\text { Buckhardt }\end{array}$ & Folha & fusiforme & verde & Glabra & Fig. 7 \\
\hline & 8 & Geraldesia $\mathrm{sp}$. & Folha & discóide & verde & Glabra & Fig. 8 \\
\hline & 9 & Cecidomyiidae & Folha & cilíndrica & marrom & Glabra & Fig. 9 \\
\hline & 10 & Cecidomyiidae & Botão apical & roseta & verde & Glabra & Fig. 10 \\
\hline & 11 & Cecidomyiidae & Ramo & esferóide & verde & Pilosa & Fig. 11 \\
\hline $\begin{array}{l}\text { Vernonia } \\
\text { polyanthes }\end{array}$ & 1 & Cecidomyiidae & $\begin{array}{l}\text { Nervura da } \\
\text { folha }\end{array}$ & esferóide & verde & Glabra & Fig. 12 \\
\hline \multirow[t]{5}{*}{ (Spreg.) Less } & 2 & Cecidomyiidae & Folha & elipsóide & verde & Pilosa & Fig. 13 \\
\hline & 3 & $\begin{array}{l}\text { Tumoplagia rudolphi Lutz } \\
\text { \& Costa Lima }\end{array}$ & Ramo & globóide & verde & Glabra & Fig. 14 \\
\hline & 4 & Cecidomyiidae & Ramo & elipsóide & verde & Glabra & Fig. 15 \\
\hline & 5 & Cecidomyiidae & Folha & discóide & verde & Pilosa & Fig. 16 \\
\hline & 6 & Cecidomyiidae & Ramo & roseta & verde & Glabra & Fig. 17 \\
\hline
\end{tabular}

a Percentagem de Ruas Pavimentadas (\%RP) teve uma relação não significativa com a abundância $(\mathrm{r}=-0.35$, n.s. $)$ e riqueza $(\mathrm{r}$ $=-0.20$, n.s. $)$ de insetos galhadores.

Morfotipos de galhas como indicadoras da qualidade ambiental. Neste estudo verificou-se que a galha da morfoespécie 5 (Figura 16), associada à planta $V$. polyanthes, ocorreu apenas no biótopo Áreas verdes urbanas, enquanto a morfo-espécie 4 (Figura 4), associada a B. dracunculifolia, ocorreu apenas nos biótopos menos impactados pela urbanização, Rural e Áreas verdes urbanas.

\section{DISCUSSÃO}

Altas temperaturas, compactação do solo, área restrita de enraizamento, variações na intensidade de luz e vento e deficiências hídricas e nutricionais são fatores que podem ocasionar estresse em plantas situadas em centros urbanos, em comparação àquelas situadas em ambientes naturais. Assim sendo, postula-se que tais plantas estariam mais susceptíveis a ataques de insetos herbívoros em áreas urbanas, devido a alterações em sua fisiologia e na qualidade para herbívoros. Essas alterações provavelmente acarretariam uma maior disponibilidade de nutrientes solúveis e uma menor produção de compostos secundários, responsáveis pela defesa da planta (Dreistadt et al. 1990).

Foi observada a tendência de ocorrência de menores valores de abundância de galhas por planta hospedeira em biótopos mais intensamente ocupados. Biótopos de áreas rurais e áreas verdes urbanas (em princípio, menos estressantes para plantas) apresentaram maior abundância de insetos galhadores. No entanto, a razão riqueza de galhas/número de plantas hospedeiras não variou nos diferentes biótopos.

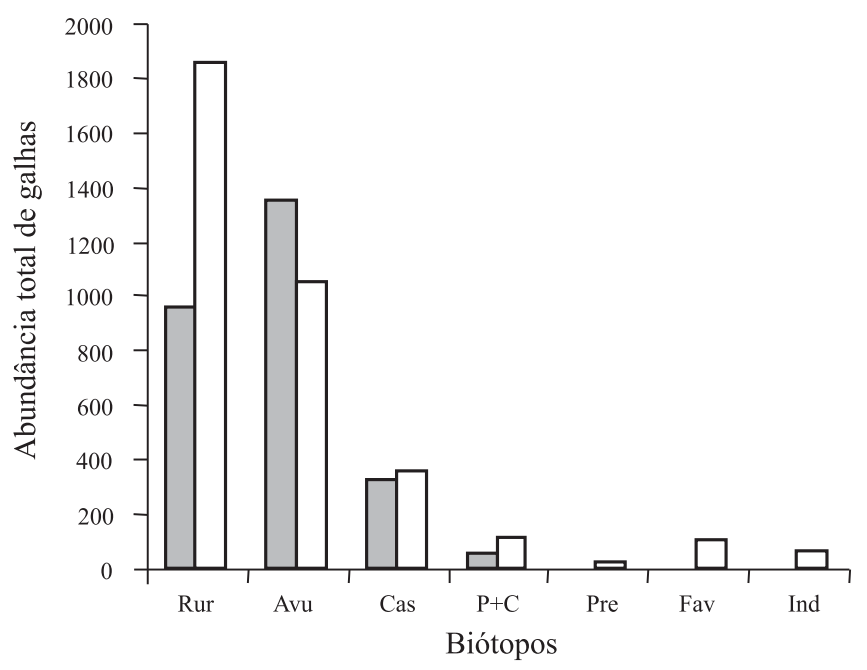

Fig. 19. Abundância total de galhas de insetos associadas a Baccharis dracunculifolia $\square$ e Vernonia polyanthes $\square$ amostradas nos biótopos urbanos de Belo Horizonte, MG. (Rur: Rural; Avu: Áreas verdes urbanas; Cas: Casas; P+C: Prédios e Casas; Pre: Prédios; Fav: Favelas e Ind: Industrial). 
Tabela III. Abundância relativa de morfo-espécies de insetos galhadores de Baccharis dracunculifolia (Asteraceae) nas localidades amostradas. (Rur: Rural; Avu: Áreas verdes urbanas; Cas: Casas; P+C: Prédios e Casas; Pre: Prédios; Fav: Favelas e Ind: Industrial)

\begin{tabular}{|c|c|c|c|c|c|c|c|c|c|c|c|c|}
\hline \multirow{2}{*}{ Localidade / Biótopo } & & \multicolumn{11}{|c|}{ Morfotipos de galhas } \\
\hline & & G 1* & G2 & G3 & G4 & G5 & G6 & G7 & G8 & G9 & G10 & G11 \\
\hline Baleia & Rur & 0 & 0.084 & 0.011 & 0.073 & 0.178 & 0 & 0.028 & 0.513 & 0.038 & 0.061 & 0.014 \\
\hline São Benedito & Rur & 0 & 0 & 0 & 0 & 0 & 0 & 0 & 0 & 0 & 0 & 0 \\
\hline Trevo & Rur & 0 & 0 & 0 & 0 & 0 & 0 & 0 & 0 & 0 & 0 & 0 \\
\hline Horto & Avu & 0.454 & 0.203 & 0.072 & 0.234 & 0 & 0.006 & 0 & 0 & 0.031 & 0 & 0.040 \\
\hline Estação.Ecológica & Avu & 0.710 & 0.028 & 0.078 & 0.009 & 0 & 0 & 0 & 0.068 & 0.074 & 0 & 0.033 \\
\hline Zoológico & Avu & 0.008 & 0 & 0.013 & 0.017 & 0 & 0.932 & 0 & 0.013 & 0.017 & 0 & 0 \\
\hline Serra & Cas & 0.044 & 0.087 & 0 & 0 & 0.087 & 0.565 & 0.087 & 0 & 0.13 & 0 & 0 \\
\hline Bandeirantes & Cas & 0.155 & 0 & 0.024 & 0 & 0.039 & 0 & 0.159 & 0.420 & 0.034 & 0.121 & 0.048 \\
\hline Castelo & Cas & 0.262 & 0.143 & 0.024 & 0 & 0.036 & 0 & 0.535 & 0 & 0 & 0 & 0 \\
\hline Prado & Cas & 0 & 0 & 0 & 0 & 0 & 0 & 0 & 0 & 0 & 0 & 0 \\
\hline Funcionários & $\mathrm{P}+\mathrm{C}$ & 0 & 0 & 0 & 0 & 0 & 0 & 0 & 0 & 0 & 0 & 0 \\
\hline Caiçara & $\mathrm{P}+\mathrm{C}$ & 0 & 0 & 0 & 0 & 0 & 0 & 0 & 0 & 0 & 0 & 0 \\
\hline Sion & $\mathrm{P}+\mathrm{C}$ & 0.125 & 0.875 & 0 & 0 & 0 & 0 & 0 & 0 & 0 & 0 & 0 \\
\hline Coração Eucarístico & $\mathrm{P}+\mathrm{C}$ & 0 & 0 & 0 & 0 & 0 & 0 & 0 & 0 & 0 & 0 & 0 \\
\hline Eldorado & Pre & 0 & 0 & 0 & 0 & 0 & 0 & 0 & 0 & 0 & 0 & 0 \\
\hline Cafezal & Fav & 0 & 0 & 0 & 0 & 0 & 0 & 0 & 0 & 0 & 0 & 0 \\
\hline Nova Pampulha & Fav & 0 & 0 & 1 & 0 & 0 & 0 & 0 & 0 & 0 & 0 & 0 \\
\hline Barreiro & Ind & 0 & 0 & 0 & 0 & 0 & 0 & 0 & 0 & 0 & 0 & 0 \\
\hline
\end{tabular}

* A letra G significa morfo-tipos de galha acompanhada por seu respectivo número

Verificou-se, ainda, uma resposta biológica diferencial por parte da fauna de insetos associados às duas espécies de plantas hospedeiras, Baccharis dracunculifolia e Vernonia polyanthes. A riqueza de galhadores de $B$. dracunculifolia apresentou variações similares ao parâmetro abundância de galhas, ocorrendo um maior número de morfo-espécies de insetos galhadores em biótopos menos urbanizados. No entanto, a riqueza de galhadores de $V$. polyanthes não acompanhou qualquer tendência, havendo similaridade entre a riqueza de galhadores tanto em áreas rurais e áreas verdes urbanas quanto em locais onde o uso e ocupação do solo são intensos (biótopos Prédios, Favelas e Industrial). Conclui-se que a comunidade de $B$. dracunculifolia mostrou-se mais sensível a modificações ambientais.

Os valores de índices de diversidade e equitabilidade específicos das duas comunidades mostram que os biótopos que apresentam maior abundância de galhas não são os mais diversos. Além disso, verificou-se que a comunidade de insetos galhadores apresentou morfo-espécies dominantes tanto em áreas rurais quanto em áreas mais intensamente ocupadas, como os biótopos Industrial e Prédios e casas. Neste estudo esperava-se que uma maior dominância de espécies ocorresse apenas em áreas mais urbanizadas. No entanto, existem relatos da dominância de determinadas espécies de insetos galhadores em comunidades naturais. A espécie Baccharopelma baccharidis Burck, 1987 (Homoptera: Psyllidae), por exemplo, é responsável por cerca de $70 \%$ das galhas (designada neste estudo como morfo-espécie 7) amostradas em $B$. dracunculifolia em comunidades naturais (Araújo et al. 1995). Dessa forma, tornam-se necessários outros estudos que comparem a dominância de espécies de insetos galhadores associados a plantas hospedeiras que tenham ocorrência tanto em áreas urbanas como em comunidades naturais, em escala temporal e espacial.

Tal situação pode ser resultante de três fatores fortemente relacionados: estrutura de habitats dentro dos biótopos, abundância / idade do recurso planta, e intensidade / freqüência de manejo de áreas livres, sejam estas parques urbanos, praças ou terrenos baldios. Neste estudo, as localidades amostradas

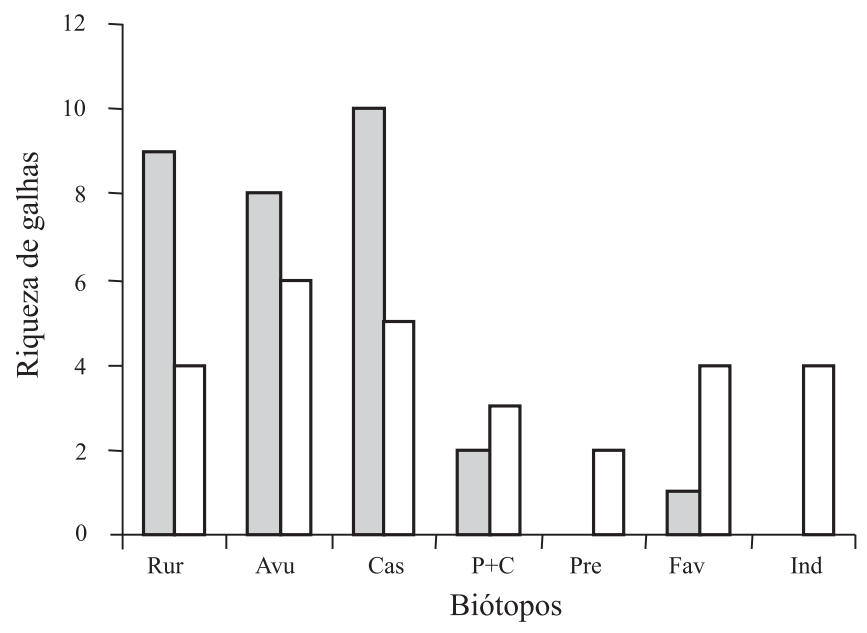

Fig. 20. Riqueza de galhas de insetos associadas a Baccharis dracunculifolia $\square$ e Vernonia polyanthes $\square$ amostradas nos biótopos urbanos de Belo Horizonte, MG. (Rur: Rural; Avu: Áreas verdes urbanas; Cas: Casas; P $+\mathrm{C}$ : Prédios e Casas; Pre: Prédios; Fav: Favelas e Ind: Industrial). 
Tabela IV. Abundância relativa de morfo-espécies de insetos galhadores de Vernonia polyanthes (Asteraceae) nas localidades amostradas. (Rur: Rural; Avu: Áreas Verdes urbanas; Cas: Casas; P+C: Prédios e Casas; Pre: Prédios; Fav: Favelas e Ind: Industrial)

\begin{tabular}{|c|c|c|c|c|c|c|c|}
\hline \multirow{2}{*}{ Localidade } & \multirow{2}{*}{ Biótopo } & \multicolumn{6}{|c|}{ Morfotipos de galhas } \\
\hline & & G1* & G2 & G3 & G4 & G5 & G6 \\
\hline Baleia & Rur & 0 & 0 & 0 & 0 & 0 & 0 \\
\hline São Benedito & Rur & 0.630 & 0 & 0.104 & 0.200 & 0 & 0.066 \\
\hline Trevo & Rur & 0.808 & 0 & 0.039 & 0.147 & 0 & 0.006 \\
\hline Horto & Avu & 0.071 & 0 & 0.211 & 0.088 & 0.403 & 0 \\
\hline Estação Ecológica & Avu & 0.530 & 0.002 & 0.167 & 0.008 & 0.096 & 0 \\
\hline Zoológico & Avu & 0.446 & 0 & 0.215 & 0.003 & 0 & 0.187 \\
\hline Serra & Cas & 0 & 0 & 0.276 & 0 & 0 & 0.724 \\
\hline Bandeirantes & Cas & 0.383 & 0.038 & 0.238 & 0.081 & 0 & 0.260 \\
\hline Castelo & Cas & 0.433 & 0 & 0.011 & 0.136 & 0 & 0.420 \\
\hline Prado & Cas & 0 & 0 & 0 & 0 & 0 & 0 \\
\hline Funcionários & $\mathrm{P}+\mathrm{C}$ & 0 & 0 & 1 & 0 & 0 & 0 \\
\hline Caiçara & $\mathrm{P}+\mathrm{C}$ & 0.571 & 0 & 0.191 & 0 & 0 & 0.238 \\
\hline Sion & $\mathrm{P}+\mathrm{C}$ & 0.764 & 0 & 0.045 & 0 & 0 & 0.191 \\
\hline Coração Eucarístico & $\mathrm{P}+\mathrm{C}$ & 0 & 0 & 0 & 0 & 0 & 0 \\
\hline Eldorado & Pre & 0.182 & 0 & 0.818 & 0 & 0 & 0 \\
\hline Cafezal & Fav & 0.022 & 0 & 0.377 & 0.066 & 0 & 0.535 \\
\hline Nova Pampulha & Fav & 0.151 & 0 & 0.547 & 0 & 0 & 0.302 \\
\hline Barreiro & Ind & 0.124 & 0 & 0.736 & 0.047 & 0 & 0.093 \\
\hline
\end{tabular}

* A letra G significa morfo-espécie de galha acompanhada por seu respectivo número

foram escolhidas de acordo com o tipo de uso e ocupação do solo. No entanto, devem ser considerados fatores como a estrutura e diversidade de habitats dentro de cada biótopo urbano, o que poderia gerar respostas específicas de determinados taxa (McIntyre et al. 2001, Frankie \& Ehler 1978), tanto de plantas hospedeiras, como dos insetos que nelas induzem galhas. Assim, áreas industriais poderiam conter habitats que fornecem condições básicas para abrigar fauna de galhadores diversa, como verificado na comunidade associada a $V$. polyanthes. Estudos mais aprofundados sobre a fauna de insetos associada a esta planta hospedeira são necessários, de forma a testar a hipótese de que áreas industriais proporcionam condições edáficas (nutrientes e umidade do solo) e climáticas diferenciadas para crescimento e desenvolvimento de plantas invasoras e colonização de insetos herbívoros. Ainda assim, seriam pertinentes questões como: 1) Em que fase do desenvolvimento da planta ocorre a colonização por insetos indutores de galhas?; 2) Existe relação entre o nível de poluição atmosférica destas áreas e a diversidade de insetos associados? 3) As galhas de insetos poderiam acumular poluentes atmosféricos e/ou resíduos químicos em seus tecidos?

Segundo Samways (1994), a diversidade de insetos em áreas urbanas está altamente relacionada com as condições e recursos disponíveis para as fases da vida do inseto. A abundância do recurso planta sofre grandes variações em centros urbanos, e a limitação na disponibilidade de habitats pode resultar em densidade e/ou abundância relativa diferencial de plantas hospedeiras de insetos galhadores (em comparação a não-hospedeiras), diminuindo assim as chances de espécies adicionais destes insetos ocorrerem (ver Root 1973; Solomon 1981, Kennedy \& Southwood 1984). Além disso, a "idade" do recurso planta, é possivelmente um dos fatores mais importantes a atuar nos padrões já observados. Frankie \& Ehler (1978) observaram que as plantas hospedeiras Quercus fusiformis e $Q$. virginiana, quando isoladas, apresentavam grandes infestações de cinipídeos galhadores, comparado a indivíduos que ocorriam em grupos ou em áreas naturais. Os autores sugeriram que as árvores isoladas haviam sido recentemente plantadas e a fauna de insetos associada havia sido removida, ocorrendo primeiramente a colonização dos galhadores e posteriormente de seus inimigos naturais. Altos níveis de infestação não foram verificados em áreas naturais, já que parasitóides controlavam a densidade de galhas. O tamanho da planta hospedeira ou quantidade de recurso disponível para o inseto galhador também apresenta relações com a "idade" do recurso planta. No caso de Baccharis dracunculifolia DC, verificou-se uma correlação muito fraca entre a abundância de galhas de Baccharopelma baccharidis Burk 1987 (Homoptera: Psyllidae) e a altura da planta hospedeira (Lara \& Fernandes 1994).

O manejo de áreas verdes em centros urbanos pode influenciar a composição de espécies de uma comunidade de insetos (McGeoch \& Chown 1997). Neste estudo, esta situação refletiu-se, por exemplo, nos resultados de diversidade e equitabilidade por biótopo urbano, onde favelas e bairro industrial apresentam valores maiores que áreas rurais. O mesmo ocorreu para o grau de similaridade da riqueza de espécies de galhadores associados a $V$. polyanthes, entre os biótopos amostrados. 
Tabela V. Valores de índice de diversidade Shannon-Winner (H)e Equitabilidade (Eh) calculados a partir da abundância e riqueza de galhas de insetos em Vernonia polyanthes e Baccharis dracunculifolia.

\begin{tabular}{lcccc}
\hline BIÓTOPO & V.polyanthes $(\mathrm{H})$ & V.polyanthes $(\mathrm{Eh})$ & B. dracunculifolia $(\mathrm{H})$ & B. dracunculifolia $(\mathrm{Eh})$ \\
\hline 1 - Rural & 0.683 & 0.493 & 1.600 & 0.695 \\
2 - Áreas verdes urbanas & 1.301 & 0.726 & 1.611 & 0.775 \\
3 - Casas & 1.354 & 0.841 & 1.914 & 0.831 \\
4 - Prédios e casas & 0.761 & 0.693 & 0.377 & 0.544 \\
5 - Prédios & 0.474 & 0.684 & $*$ & $*$ \\
6 - Favelas & 1.047 & 0.755 & 0.000 & - \\
7 - Industrial & 0.852 & 0.615 & $*$ & $*$ \\
\hline
\end{tabular}

Obs: Os asteríscos indicam que não foram encontradas galhas de insetos nas plantas hospedeiras amostradas no dado biótopo.

O grau de permeabilidade do solo, indicado por valores de Porcentagem de Ruas Pavimentadas em cada localidade, não mostrou relação com a riqueza e abundância de galhas. No entanto, a Porcentagem de Cobertura Vegetal apresentou relação positiva e forte com a abundância e riqueza de insetos galhadores. Estes resultados sugerem que a permeabilidade do solo não foi fator preponderante na variação das comunidades de galhadores associadas a plantas invasoras de áreas urbanas e peri-urbanas.

Contrariamente, áreas verdes urbanas, fazendas, parques recreativos, terrenos baldios, praças, jardins públicos e privados, margens de rodovias e ferrovias atuam como "corredores biológicos", conectando habitats urbanos com habitats de vegetação natural e agrícolas, permitindo o estabelecimento e manutenção da diversidade de insetos (Zapparoli 1997). Desta forma, fica evidente a importância da implantação e conservação de áreas verdes em ambientes urbanos e peri-urbanos para a manutenção da diversidade de insetos, como constatado neste estudo.

Verificou-se, ainda, que determinados morfotipos de galhas de insetos (Figuras 4 e 16) ocorreram exclusivamente em áreas sob menor impacto de urbanização, indicando a sensibilidade de insetos galhadores a alterações ambientais, sejam elas em manchas de vegetação nativa ou centros urbanos. Além disso, devido à sua especificidade, galhas de insetos têm sido utilizadas como item chave na identificação intra e interespecífica de plantas hospedeiras (Floate et al. 1996).

Brown Jr. (1991) classificou grupos de insetos (Odonata, Isoptera, Hymenoptera, Lepidoptera) usualmente utilizados no monitoramento ambiental, baseando-se em onze critérios de qualidades desejáveis para grupos bioindicadores. Apesar de insetos galhadores não estarem incluídos neste trabalho, tais organismos correspondem muito bem aos requisitos propostos pelo autor. Estes insetos são taxonomicamente e ecologicamente diversificados (Fernandes et al. 2001, Julião et al. 2002), possuem alta fidelidade ecológica, são fáceis de encontrar em campo (Fernandes \& Price 1988, Lara \& Fernandes 1996), possuem relações específicas com outras espécies e recursos (Larson 1998), são bem estudados (Mani 1964, Gagné 1994) e possibilitam obtenção de grandes amostras (6.226 galhas de insetos em biótopos deste estudo). Além disso, as galhas de insetos são estruturas persistentes que podem auxiliar na identificação de espécies de plantas hospedeiras a partir de sua morfologia externa (forma, cor, pubescência, tipo de ocorrência) uma vez que diferentes espécies de insetos galhadores induzem galhas morfologicamente distintas (veja Ribeiro et al. 1999).

Dessa forma, estudos implementando testes da aplicabilidade de tais organismos como bioindicadores se tornam imprescindíveis. Detalhes sobre a distribuição das morfoespécies de galhadores associadas a $B$. dracunculifolia e $V$. polyanthes poderão contribuir com resultados mais consistentes sobre qualidade ambiental em áreas urbanas e peri-urbanas. Além disso, estudos em outras metrópoles permitirão a validação das informações obtidas neste estudo, bem como a obtenção de padrões para comunidades de galhadores em ambientes urbanos.

Agradecimentos. Agradecemos ao Programa RHAE/CNPq pelo financiamento parcial do projeto original "Mapeamento de Biótopos em Áreas Urbanas", a Brandt Meio Ambiente, a D. L. Silvano pelo auxílio no campo, M.A. Ferreira pela elaboração das figuras, e ao CNPq (521772/95-7).

\section{REFERÊNCIAS}

Andrade, G. I.; I. M. Silva; G. W. Fernandes \& V. L. Scatena. 1995. Aspectos biológicos das galhas de Tomoplagia rudolphis (Diptera: Tephritidae) em Vernonia polyanthes (Asteraceae). Revista Brasileira de Biologia 55: 819-829.

Araújo A. M.; G. W. Fernandes, L. C. Bedê. 1995. Influência do sexo e fenologia de Baccharis dracunculifolia DC. (Asteraceae) sobre insetos herbívoros. Revista Brasileira de Entomologia 39: 347 353.

Bagatto, G. \& J. D. Shorthouse. 1991. Accumulation of copper and nickel in plant tissues and an insect gall of lowbush blueberry, Vaccinium angustifolium, near an ore smelter at Sudbury, Ontario, Canada. Canadian Journal of Botany 69: 1483-1490.

Bedê, L. C.; M. Weber; S. Resende; W. Piper \& W. Schulte. 1997. Manual para mapeamento de biótopos no Brasil: base para um planejamento ambiental eficiente. Fundação Alexander Brandt, $2^{a}$ edição, Belo Horizonte.

Boldt, O. E. 1989. Baccharis (Asteraceae), a review of its taxonomy, phytochemistry, ecology, economic status, natural enemies and the potencial for its biological control in the United States. Temple, Texas: United States Department of Agriculture, 
Agricultural Research Service Grassland, Soil and Water Research Laboratory, $39 \mathrm{pp}$.

Brown Jr., K. S. 1991. Conservation of neotropical environments: Insects as indicators. In N.M. Collins and J.A. Thomas (eds), The Conservation of Insects and Their Habitats. $15^{\text {th }}$ Symposium of the Royal Entomological Society of London, 14-15 September 1986, at the Dep. of Physics Lecture Theatre, Imperial College, London. Academic Press, London. pp. 349-404.

Disney, R. H. L. 1986. Assessments using invertebrates: posing the problem. In M.B. Usher (ed), Wildlife Conservation Evaluation. Chapman and Hall: London. p. 271-293.

Dreistadt, S. H.; D. L. Dahlsten \& G. W. Frankie. 1990. Urban forest and insect ecology: complex interactions among trees, insects, and people. BioScience 40: 192-198.

Espírito-Santo, M. M. \& G. W. Fernandes. 1998. Abundance of Neopelma baccharidis (Homoptera: Psyllidae) galls on the dioecious shrub Baccharis dracunculifolia (Asteraceae). Environmental Entomology 27: 870-876.

Fernandes, G. W. \& P. W. Price. 1988. Biogeographical gradients in galling species richness: tests of hypotheses. Oecologia 76: 161167.

Fernandes, G. W.; A. S. de Paula \& R. Loyola Jr. 1995. Distribuição diferencial de insetos galhadores entre habitats e seu possível uso como bioindicadores. Vida Silvestre Neotropical 4: 133-139.

Fernandes, G. W.; M. A. A. Carneiro; A. C. F. Lara; L. R. Allain; G. I. Andrade; G. R. Julião; T. R. Reis \& I. M. Silva. 1996. Galling insects on neotropical species of Baccharis (Asteraceae). Tropical Zoology 9: 315-332.

Fernandes, G. W.; G. R. Julião; R. Araújo; S. Araújo; J. A. Lombardi; D. Negreiros \& M. A. A. Carneiro. 2001. Distribution and morphology of insect galls of the rio Doce valley, Brazil. Naturalia 26: 211244

Floate, K. D.; G. W. Fernandes \& J. A. Nilsson. 1996. Distinguishing intrapopulational categories of plants by their insect faunas: galls on rabbitbrush. Oecologia 105: 221-229.

Frankie, G. W. \& L. E. Ehler. 1978. Ecology of insects in urban environments. Annual Review of Entomology 23: 367-387.

Gagné, R. J. 1994. The gall midges of the neotropical region. Ithaca, NY: Comstock, $352 \mathrm{pp}$.

Julião, G. R.; M. E. C. Amaral \& G. W. Fernandes. 2002. Galhas de insetos e suas plantas hospedeiras do Pantanal sul-mato-grossense. Naturalia 27: 47-74.

Kennedy, C. E. J. \& T. R. E. Southwood. 1984. The number of species of insects associated with British trees: a re-analysis. Journal of Animal Ecology 53: 455-478.

Lara, A. C. F. \& G. W. Fernandes. 1994. Distribuição de galhas de Neopelma baccharidis (Homoptera: Psyllidae) em Baccharis dracunculifolia (Asteraceae). Revista Brasileira de Biologia 54: $661-668$.

Lara, A. C. F. \& G. W. Fernandes. 1996. The highest diversity of galling insects: Serra do Cipó, Brazil. Biodiversity Letters 3: 111-114.

Larson, K. C. 1988. The impact of two gall-forming arthropods on the photosynthetic rates of their hosts. Oecologia 115: 161-166.

Leitão Filho, H. F.; C. Aranha \& O. Bacchi. 1972. Plantas invasoras de culturas no estado de São Paulo. São Paulo, Hucitec.

Mani, M. S. 1964. The ecology of plant galls. The Hague: Junk, 640 pp.
McDonnell, M. J. \& S. T. A. Pickett. 1990. Ecosystem structure and function along urban-rural gradient: an unexploited opportunity for ecology. Ecology 71: 1232-1237.

McGeoch, M. A. \& S. L. Chown. 1997. Impact of urbanization on a gall-inhabiting Lepidoptera assemblage: the importance of reserves in urban areas. Biodiversity and Conservation 6: 979-993.

McIntyre, N. E.; J. Rango; W. F. Fagan \& S. H. Faeth. 2001. Ground arthropod community structure in a heterogeneous urban environment. Landscape and Urban Planning 52: 257-274.

Oliver, I. \& A. J. Beattie. 1996. Invertebrate morphospecies as surrogates for species: a case study. Conservation Biology 10: 99-109.

Oliver, I.; A. J. Beattie \& A. York. 1998. Spatial fidelity of plant, vertebrate and invertebrate assemblages in multiple-use forest in eastern Australia. Conservation Biology 12: 822-835.

Pik, A. J.; I. Oliver \& A. J. Beattie. 1999. Taxomic sufficiency in ecological studies of terrestrial invertebrates. Australian Journal of Ecology 24: 555-562.

Prefeitura de Belo Horizonte. 1994. Urbanismo e Obras / Estatísticas e Informações. URL: http://www.pbh.gov.br

Price, P. W.; G. W. Fernandes; A. C. F. Lara; J. Brawn; H. Barrios; M. G. Wright; S. P. Ribeiro \& N. Rothcliff. 1998. Global patterns in local number of insect galling species. Journal of Biogeography 25: 581-591.

Ribeiro, J. E. L. S.; M. J. G. Hopkins; A. Vicentini; C. A. Sothers; M. A. S. Costa; J. M. Brito; M. A. D. Souza; L. G. P. Martins; L. G. Lohmann; P. A. C. L. Assunção; E. C. Pereira; C. F. Silva; M. R. Mesquita; L. C. Procópio. 1999. Flora da Reserva Ducke: guia de identificação das plantas vasculares de uma floresta de terrafirme na Amazônia Central. DFID, INPA, Manaus.

Root, R. B. 1973. Organization of a plant-arthropod association in simple and diverse habitats: the fauna of colards (Brassica oleracea). Ecological Monographs 43: 95-124.

Samways, M. J. 1994. Insect conservation biology. Chapman \& Hall: London.

Samways, M. J. \& N. S. Steytler. 1996. Dragonfly (Odonata) distribution patterns in urban and forest landscapes and recommendations for riparian management. Biological Conservation 78: 279-288.

Silva, I. M.; G. I. Andrade; G. W. Fernandes \& J. P. Lemos-Filho. 1996. Parasitic relationship between a gall-forming insect Tomoplagia rudolphi (Diptera: Tephritidae) and its host plant (Vernonia polyanthes, Asteraceae). Annals of Botany 78: 45-48.

Solomon, B. P. 1981. Response of a host specific herbivore to resource density, relative abundance and phenology. Ecology 62: 12051214.

Sukopp, H. \& S. Weiler. 1988. Biotope mapping and nature conservation strategies in urban areas of the Federal Republic of Germany. Landscape and Urban Planning 15: 39-58.

Taylor, R. J. \& N. Doran. 2001. Use of terrestrial invertebrates as indicators of the ecological sustainability of forest management under the Montreal Process. Journal of Insect Conservation 5: 221-231.

Zapparoli, M. 1997. Urban development and insect biodiversity of the Rome area, Italy. Landscape and Urban Planning 38: 77-86.

Zar, J. H. 1996. Biostatistical Analysis (3 ${ }^{\text {rd }}$ edn.). Prentice-Hall, New Jersey.

Recebido em 16.V.2003; aceito em 22.XI.2004 